\title{
Propagación asexual de especies endémicas y amenazadas del género Passiflora en los Andes colombianos
}

\author{
Asexual propagation of endemic and threatened species \\ of the genus Passiflora in the Colombian Andes \\ Blanca Luz Caleño-Ruíz ${ }^{\circledR}{ }^{\circledR}$, Gustavo Morales-Liscano ${ }^{2}$
}

Caleño-Ruíz, B. L. y Morales-Liscano, G. (2019). Propagación asexual de especies endémicas y amenazadas del género Passiflora en los Andes colombianos. Colombia Forestal, 22(2), 67-82.

Recepción: 12 de enero de 2019

\section{Resumen}

El género Passiflora tiene una gran representatividad en Colombia e incluye especies endémicas y amenazadas distribuidas principalmente en los Andes. Es necesario fomentar el conocimiento de estas especies y prácticas adecuadas para su conservación debido a que representan fuentes de germoplasma valiosos. Evaluamos el efecto de un tratamiento natural y uno sintético sobre el desempeño de estacas de cinco especies de Passiflora endémicas y amenazadas de los Andes colombianos. Ambos tratamientos promovieron la producción de raíces y el tratamiento sintético tuvo los mayores valores y una menor mortalidad. La respuesta de las estacas fue diferente entre las cinco especies y las variables medidas tuvieron valores distintos para cada una durante el desarrollo posterior a la siembra. Ambos tratamientos mejoran el desarrollo radicular de estacas de Passiflora, aunque el tratamiento sintético tuvo ventajas sobre el natural; sin embargo, su empleo debe estar orientado a las características de desarrollo de cada especie.

Palabras clave: ácido naftalenacético, estaca, hormona vegetal, sábila, tejido aéreo, tejido radicular.
Aprobación: 4 de junio de 2019

\begin{abstract}
The Passiflora genus has a large representativeness in Colombia and includes endemic and threatened species distributed mainly in the Andes. It is necessary to promote knowledge of these species and appropriate practices for their conservation because they represent valuable sources of germplasm. We evaluated the effect of a natural and a synthetic treatment on the performance of stakes of five endemics and threatened species of Passiflora from the Colombian Andes. Both treatments promoted the production of roots and the synthetic treatment had the highest values and a lower mortality. The response of the stakes was different among the five species and the measured variables had different values for each one during the development after sowing. Both treatments improve the root development of Passiflora stakes, although the synthetic treatment had advantages over the natural one; however, its use must be oriented to the development characteristics of each species.
\end{abstract}

Key Words: naphthaleneacetic acid, stake, plant hormone, aloe, aerial tissue, root tissue.

Jardín Botánico de Bogotá José Celestino Mutis. Bogotá, Colombia. Correo electrónico: blcalenor@correo.udistrital.edu.co, blanca.caleno@ gmail.com. Autor para correspondencia

Jardín Botánico de Bogotá José Celestino Mutis. Bogotá, Colombia. Correo electrónico: gustavmorales@gmail.com. 


\section{INTRODUCCIÓN}

El género Passiflora es de los más diversos dentro de la familia Passifloraceae, exclusivo de América y con cerca de 450 especies registradas en el trópico americano (Ulmer y MacDougal, 2004). Es el género más importante a nivel económico dentro de la familia debido a que muchas de las especies se cultivan para el consumo de frutos, para usos medicinales o como ornamentales gracias a la belleza y morfología de su flor, de la cual viene su nombre común "flor de la pasión" debido a la posición de los estambres que recuerdan la pasión de cristo (Killip, 1938; Dhawan, Dhawan y Sharma, 2004; Ulmer y MacDougal, 2004; Yockteng, D’Eeckenbrugge y Souza-Chies, 2011). En Colombia, este género es también el más representativo de la familia con 162 de las 167 especies registradas para 2007, de las cuales 58 son endémicas de Colombia (Ocampo et al., 2007). Para el 2015, los registros de Bernal, Gradstein y Celis (2015) señalaron 163 especies de Passiflora de un total de 187 para la familia Passifloraceae en Colombia.

La región andina colombiana es considerada como la más diversa en especies de Passifloraceae, especialmente los departamentos de Antioquia, Valle del Cauca, Cundinamarca y Santander, los cuales también abarcan el área con mayor endemismo (Ocampo et al., 2007). Incluso, se reconoce a los Andes del norte de Colombia y Ecuador como el centro de diversidad del género Passiflora debido a la diversidad de hábitats de altas elevaciones (Ocampo et al., 2007; Ocampo, d'Eeckenbrugge y Jarvis, 2010; Yockteng et al., 2011). Sin embargo, esta región ha sufrido un proceso de recuperación y transformación continua (Rubiano et al., 2017) y aunque actualmente las mayores tasas de deforestación se concentran en otras regiones del país (Ideam, 2018), se ha demostrado que los ecosistemas andinos enfrentaron en el pasado altas tasas de deforestación (Armenteras y Rodríguez, 2014). Esto último ha sido un factor fundamental para la distribución actual de Passiflora y la reducción de sus hábitats, situación por la que actualmente 29 de las 163 especies (47.3 \%) está bajo alguna categoría de amenaza (Bernal et al., 2015) y en su mayoría fuera de áreas protegidas (Ocampo et al., 2007, 2010).

Las especies de Passiflora no solo tienen importancia ornamental, sino también un gran potencial fitofarmacéutico debido a los alcaloides, fenoles, flavonoides y compuestos cianogénicos que han sido reportados para el género y que tienen propiedades sedativas, vermífugas, ansiolíticas, antiespasmódicas, diuréticas y eméticas (Dhawan et al., 2004; Yockteng et al., 2011). Además, son fuente de germoplasma para la mejora de especies cultivadas como el maracuyá (Passiflora edulis f. flavicarpa O. Deg.) o la gulupa (Passiflora edulis Sims var. edulis) que, entre otros usos, tienen las mayores aplicaciones clínicas en el mundo (Dhawan et al., 2004; Yockteng et al., 2011). Para Colombia, se han reportado 22 especies de las que se consumen los frutos, de las cuales 9 son comercialmente cultivadas (Ocampo et al., 2007).

A pesar de su amplia importancia, el conocimiento sobre estas especies es escaso pero necesario para, entre otras cosas, conservar su germoplasma con el fin mejorar la producción agrícola, preservar el ambiente y los organismos que de ellas dependen (Yockteng et al., 2011). Es por esta razón que las estrategias de conservación ex situ resultan indispensables para cumplir con este objetivo. Mundialmente, existen más de 2000 accesiones de germoplasma que representan cerca de 159 especies de Passiflora conservadas (Faleiro, Junqueira y Braga, 2005; Yockteng et al., 2011) que, sumadas a los aportes en colecciones vivas de jardines botánicos, instituciones académicas, sociedades de plantas y aficionados, son un gran aporte para lograr su conservación (Yockteng et al., 2011). Sin embargo, muchas de estas colecciones necesitan diversificación e inclusión de más individuos y especies silvestres preferiblemente amenazadas para garantizar variabilidad genética (Yockteng et al., 2011; Castro, Oliveira, Jesus, Soares y Margarido, 2016), además de mayores 
estudios en ecología, fitoquímica y genética de las mismas (Yockteng et al., 2011).

Por otro lado, existe un avance considerable en la propagación de algunas especies de Passiflora que se enfoca principalmente en la producción a escala comercial (Parra, Carranza, Cárdenas y Miranda, 2010; Ocampo y Wyckhuys, 2012; Ozarowski y Thiem, 2013), pero muy poco en el desarrollo de técnicas de propagación que contribuyan a la conservación de estas especies (Pacheco et al., 2016). Además, en muchos casos la información disponible acerca de ecología, fitoquímica, genética, propagación y manejo se concentran particularmente en $P$. edulis (Yockteng et al., 2011; Ocampo y Wyckhuys, 2012), lo que significa un aporte importante, pero que debe servir como referente para estudiar otras especies, en especial si son silvestres, endémicas o amenazadas, pues son estos taxones los que necesitan ser conocidos y conservados (Yockteng et al., 2011).

De esta manera, uno de los propósitos de las colecciones especializadas para la conservación (Cеpac), de la línea de colecciones vivas del Jardín Botánico de Bogotá José Celestino Mutis (JBB), es contribuir a la conservación ex situ de especies vegetales a través de la preservación e investigación (Resolución N. 249, 2018). Estas colecciones incluyen especies de la familia Passifloraceae que han sido priorizadas de acuerdo con su distribución geográfica y altitudinal particular, además de su representatividad para el país por su grado de endemismo, estado de amenaza y usos potenciales. Así pues, es un deber para el JBB aumentar la representatividad de especies y realizar investigación en este tipo de colecciones con el fin de fortalecer su estrategia de conservación ex situ (Samper y García, 2001; García, Moreno, Londoño y Sofrony, 2010).

En ese sentido, para las colecciones vivas del JBB se requiere información relacionada con la propagación de especies que constantemente se traen de campo con el fin hacer más exitoso el proceso de enriquecimiento. Además, es de gran importancia conocer las técnicas más apropiadas para la propagación de estacas de Passiflora, pues es el propágulo más fácil de encontrar en las expediciones que realiza la entidad, ya que no siempre se localizan frutos para obtener semillas.

Para acelerar el enraizamiento de estacas provenientes de individuos silvestres y promover el éxito de sobrevivencia de este material, se aplican comúnmente las hormonas sintéticas ácido indolbutírico (AIB) y ácido naftalenacético (ANA) (Pérez, Reynel y Manta, 2002; Oliva-Cruz, 2005; Minchala-Patiño et al., 2013; Morillo et al., 2016) en la parte inferior de las estacas previo a la siembra en sustrato. Sin embargo, se ha comprobado que sustancias naturales como el extracto de sábila (Aloe vera (L.) Burm.f.) tienen efecto exitoso en el desarrollo de raíces de las estacas (Rodríguez y Hechevarría, 2004; Giraldo, Ríos y Polanco, 2009; Borges, León, Marturet y Barrios, 2016). Estos tratamientos constituyen herramientas importantes para acelerar el crecimiento de estacas de Passiflora. De esta manera, los objetivos planteados en esta investigación consisten en: 1) identificar el efecto de un tratamiento sintético (ANA) y uno natural (cristales de $A$. vera) sobre el desempeño de estacas de especies de Passiflora endémicas y amenazadas obtenidas en poblaciones silvestres; y 2) caracterizar el desarrollo de las estacas de cada especie, posterior a la siembra, con el fin de generar recomendaciones adecuadas para el manejo de cada una durante el proceso de crecimiento, de tal manera que se incremente la probabilidad de éxito en el establecimiento y conservación ex situ.

\section{MATERIALES Y MÉTODOS}

\section{Área de estudio}

El montaje experimental fue realizado en el vivero del JBB, ubicado en la localidad de Engativá (Bogotá, Colombia), con una altitud de 2650 $m$ y temperaturas que al interior del vivero alcanzan $22^{\circ} \mathrm{C}$. El muestreo y recolección de material vegetal de las especies de Passiflora endémicas y 
amenazadas fue realizado en los municipios Fosca, San Francisco y Zipacón en el departamento de Cundinamarca. Los sitios muestreados se encontraban entre los 1600 y $2500 \mathrm{~m}$ de altitud y 19 a $24^{\circ} \mathrm{C}$ de temperatura media y contaban con una vegetación transformada dentro de fincas y aledaña a carreteras y fuentes de agua (tabla 1). Para cada especie muestreada se tomaron ejemplares botánicos que fueron depositados en el Herbario del JBB.

\section{Especies estudiadas}

De acuerdo con las especies de Passiflora registradas para Colombia, su estado de conservación y endemismo (Ocampo et al., 2007; Bernal et al., 2015), así como las especies de la familia Passifloraceae que actualmente se conservan dentro de las colecciones Cepac del JBB, se priorizaron cinco especies con el fin de enriquecer la colección Cepac y contribuir a la conservación ex situ de especies de Passiflora amenazadas y endémicas del país. Tres de estas especies se encuentran catalogadas como vulnerables, de acuerdo con las categorías de la Unión Internacional para la Conservación de la Naturaleza y los Recursos Naturales (UICN), estas son: P. dawei, P. erythrophylla y $P$. pennellii, y dos de ellas catalogadas como de preocupación menor y endémicas de Colombia: P. longipes y P. smithii. Las cinco especies se encuentran dentro de un intervalo altitudinal entre 500 (P. smithii) y 3500 (P. longipes) $\mathrm{m}$ de altitud (tabla 1), principalmente sobre la cordillera oriental de los Andes (Ocampo et al., 2007; Bernal et al., 2015).

\section{Muestreo en campo y recolección del material vegetal}

Una vez fueron identificados los municipios en los cuales se encontraban las poblaciones de las especies priorizadas, se realizaron recorridos en las zonas rurales con el propósito de buscar las especies en sitios generalmente cercanos a corrientes de agua y bordes de carretera. Se seleccionaron individuos maduros que tuvieran tallos abundantes y se procedió a cosechar tallos que sumaran cerca de $6 \mathrm{~m}$ de longitud, suficientes para garantizar la cantidad de estacas necesaria. Estos tallos fueron cubiertos con periódico humedecido con agua y almacenados en bolsas plásticas con el fin de mantener su turgencia en campo. Posteriormente, en el vivero del JBB se cortaron estacas entre 15 y $20 \mathrm{~cm}$ de longitud, según la especie, y se dejaron como mínimo dos nudos por estaca (Otahola y Vidal, 2010). Al final se obtuvo un total de 180 estacas (36 por especie) a las cuales se les cortó dos tercios de cada hoja, cuando esta estaba presente, con el fin de evitar exceso de evapotranspiración (Hartmann y Kester, 1991).

\section{Diseño experimental}

Para el ensayo se emplearon dos tratamientos comúnmente empleados en el área de propagación del JBB: ácido naftalenacético al $0.4 \%$ (ANA) como tratamiento sintético y extracto de cristales de sábila $(A$. vera) como tratamiento natural; también se usaron estacas que no recibieron ninguna aplicación, las cuales fueron consideradas como testigos. Se introdujo la parte basal de 60 estacas

Tabla 1. Especies estudiadas, municipio de localización y características climáticas

\begin{tabular}{lccc}
\hline \multicolumn{1}{c}{ Especie } & Municipio & Altitud (m) & Temperatura $\left({ }^{\circ} \mathbf{C}\right)$ \\
\hline $\begin{array}{l}\text { Passiflora pennellii Killip } \\
\text { Passiflora dawei Killip }\end{array}$ & Fosca & 2000 & 22 \\
$\begin{array}{l}\text { Passiflora smithii Killip. } \\
\text { Passiflora erythrophylla Mast. }\end{array}$ & San Francisco & 1600 & 24 \\
$\begin{array}{l}\text { Passiflora longipes Juss. } \\
\text { Zipacón }\end{array}$ & 2500 & 19 \\
\hline
\end{tabular}


dentro de una solución con el respectivo tratamiento (ANA o cristal de sábila) por espacio de 20 minutos. Como sustrato, se empleó una mezcla de tierra negra cernida con cascarilla de arroz cruda en una proporción de 3:1; el sustrato resultante fue desinfectado con el método de pasteurización que consiste en humedecer el sustrato y someterlo a una temperatura de $60^{\circ} \mathrm{C}$ al interior de un horno durante una hora (Hartmann y Kester, 1991). Las estacas fueron sembradas en bolsas de 12 × $25 \mathrm{~cm}$ introduciendo una tercera parte de la estaca dentro del sustrato. Para cubrir todas las bolsas en las que fueron sembradas las estacas, se emplearon plásticos transparentes que fueron sellados a modo de cámara húmeda a lo largo del ensayo con el fin de aumentar la humedad relativa. Finalmente, los tratamientos se distribuyeron en un arreglo factorial de bloques completos aleatorizados (Montgomery, 2004) con cuatro bloques, 15 tratamientos y tres estacas por unidad experimental.

\section{Variables medidas}

Tras 19 semanas desde el inicio del ensayo, se procedió a medir en cada una de las estacas el número total de raíces, el número de ramificaciones en una muestra aleatoria de raíz de $3 \mathrm{~cm}$ de longitud, la longitud de la raíz más larga $(\mathrm{cm})$, el número de brotes, la longitud del brote más largo $(\mathrm{cm})$, el número de hojas, el número de zarcillos y el porcentaje de sobrevivencia y mortalidad.

\section{Análisis de datos}

Se realizaron pruebas de normalidad y homogeneidad de varianza empleando las pruebas de Kolmogorov-Smirnov y Barlett del paquete stats ( $R$ Core Team, 2018) y Levene del paquete car (Fox y Weisberg, 2011). Posteriormente, se realizaron pruebas de comparación Kruskal-Wallis del paquete stats (R Core Team, 2018) para evaluar la sobrevivencia y mortalidad entre especies y tratamientos. Con el fin de determinar si los tratamientos generaron efectos en el desarrollo de estacas de cada una de las especies de Passiflora evaluadas, se realizó un análisis de varianza no paramétrico de dos factores con la prueba Scheirer-Ray-Hare del paquete rcompanion (Mangiafico, 2018). Para determinar cuáles tratamientos generaron efectos sobre el crecimiento de Passiflora y cuáles de ellas presentaron respuestas distintas, se realizó una prueba post-hoc Dunn del paquete FSA (Ogle, Wheeler y Dinno, 2018). Adicionalmente, se estudiaron las correlaciones entre las variables evaluadas en las estacas con el método de Spearman del paquete Hmisc (Harrell y Dupont, 2018) y se elaboró un análisis de componentes principales (ACP) del paquete FactoMineR (Lê, Josse y Husson, 2008) con el fin de caracterizar el desarrollo de cada especie. Los análisis fueron realizados con el software estadístico R versión 3.5.1. (R Core Team, 2018).

\section{RESULTADOS}

\section{Efecto de los tratamientos sobre las especies de Passiflora}

No se encontraron diferencias entre el porcentaje de sobrevivencia y mortalidad de las estacas entre las especies (sobrevivencia K-W $x^{2}: 4, p$-valor: 0.406; mortalidad K-W $x^{2}: 4$, p-valor: 0.406) o entre los tratamientos (sobrevivencia K-W $x^{2}: 2, p$-valor: 0.368; mortalidad K-W $x^{2}: 2, p$-valor: 0.368). Sin embargo, las estacas con mayor mortalidad correspondieron a las especies $P$. dawei y $P$. smithii y aquellas que fueron tratadas con cristales de sábila (tabla 2).

Para las variables medidas en las estacas de las cinco especies de Passiflora, los tratamientos tuvieron un efecto diferenciado solamente en el número de raíces y en el número de hojas (tabla 3). Adicionalmente, el efecto de los tratamientos no fue distinto para las cinco especies evaluadas ya que las interacciones entre estos factores no fueron significativas, pero para todas las variables medidas en las estacas, la especie generó una respuesta diferenciada (tabla 3). 
Tabla 2. Sobrevivencia y mortalidad de estacas de Passiflora por especie y tratamiento.

\begin{tabular}{lcccc}
\hline & $\begin{array}{c}\text { Número de estacas } \\
\text { muertas }\end{array}$ & $\begin{array}{c}\text { Número de } \\
\text { estacas vivas }\end{array}$ & Mortalidad (\%) & $\begin{array}{c}\text { Sobrevivencia } \\
(\%)\end{array}$ \\
\hline Especie & & & & \\
P. dawei & 11 & 25 & 30.556 & 69.444 \\
P. erythrophylla & 7 & 29 & 19.444 & 80.556 \\
P. longipes & 8 & 28 & 22.222 & 77.778 \\
P. pennellii & 8 & 28 & 22.222 & 77.778 \\
P. smithii & 18 & 18 & 50.000 & 50.000 \\
Tratamiento & & & & \\
Cristal de sábila & 21 & 39 & 35.000 & 65.000 \\
ANA & 14 & 46 & 23.333 & 76.667 \\
Testigo & 17 & 43 & 28.333 & 71.667 \\
\hline
\end{tabular}

Tabla 3. Resultados de la prueba Scheirer-Ray-Hare para las variables medidas sobre las estacas de Passiflora. gl: grados de libertad, SC: suma de cuadrados, $\mathrm{H}$ : valor para el estadístico de prueba. En negrilla los valores significativos a un nivel de 0.05 .

\begin{tabular}{|c|c|c|c|c|}
\hline Factor & gl & SC & $\mathbf{H}$ & p-valor \\
\hline \multicolumn{5}{|l|}{ Número de raíces } \\
\hline Tratamiento & 2 & 9671 & 7.039 & 0.030 \\
\hline Especie & 4 & 49927 & 36.337 & $<0.001$ \\
\hline Tratamiento*Especie & 8 & 11884 & 8.649 & 0.373 \\
\hline \multirow{2}{*}{\multicolumn{5}{|c|}{ Número de ramificaciones }} \\
\hline & & & & \\
\hline Tratamiento & 2 & 2407 & 1.775 & 0.412 \\
\hline Especie & 4 & 18995 & 14.004 & 0.007 \\
\hline Tratamiento*Especie & 8 & 7570 & 5.581 & 0.694 \\
\hline Residuales & 113 & 143291 & & \\
\hline \multicolumn{5}{|c|}{ Longitud de la raíz más larga $(\mathrm{cm})$} \\
\hline Tratamiento & 2 & 48 & 0.035 & 0.983 \\
\hline Especie & 4 & 50289 & 36.565 & $<0.001$ \\
\hline Tratamiento*Especie & 8 & 8517 & 6.193 & 0.626 \\
\hline Residuales & 113 & 115813 & & \\
\hline \multicolumn{5}{|l|}{ Número de brotes } \\
\hline Tratamiento & 2 & 2292 & 24.855 & 0.289 \\
\hline Especie & 4 & 11091 & 12.024 & 0.017 \\
\hline Tratamiento*Especie & 8 & 11180 & 12.122 & 0.146 \\
\hline Residuales & 113 & 92573 & & \\
\hline \multicolumn{5}{|c|}{ Longitud del brote más largo $(\mathrm{cm})$} \\
\hline Tratamiento & 2 & 4673 & 3.397 & 0.183 \\
\hline Especie & 4 & 103096 & 74.948 & $<0.001$ \\
\hline Tratamiento*Especie & 8 & 2395 & 1.741 & 0.988 \\
\hline Residuales & 113 & 64534 & & \\
\hline \multicolumn{5}{|l|}{ Número de hojas } \\
\hline Tratamiento & 2 & 8759 & 6.405 & 0.041 \\
\hline Especie & 4 & 77587 & 56.738 & $<0.001$ \\
\hline Tratamiento*Especie & 8 & 4950 & 3.620 & 0.890 \\
\hline Residuales & 113 & 82372 & & \\
\hline \multicolumn{5}{|l|}{ Número de zarcillos } \\
\hline Tratamiento & 2 & 552 & 0.422 & 0.810 \\
\hline Especie & 4 & 88408 & 67.650 & $<0.001$ \\
\hline Tratamiento*Especie & 8 & 4542 & 3.475 & 0.901 \\
\hline Residuales & 113 & 72467 & & \\
\hline
\end{tabular}


Aunque no hubo diferencias significativas entre los tratamientos, el número de raíces por estaca fue mayor cuando se aplicó ANA en comparación con la aplicación de cristales de sábila y el testigo, y no hubo una diferencia significativa en el número de raíces por estaca para las que fueron tratadas con cristales de sábila (Aloe vera) y las testigos (figura 1a). En cuanto a la especie, $P$ dawei y $P$ erytrophylla reportaron las producciones más altas de raíces en comparación con $P$. longipes, la cual presentó la menor cantidad de raíces (figura 1 b).

El número de hojas fue similar entre los diferentes tratamientos, pero las estacas tratadas con cristales de sábila presentaron el mayor número de hojas, seguidas por las estacas testigo y las que fueron tratadas con ANA (figura 1c). En cuanto a especies, las estacas de $P$. longipes presentaron un número de hojas mayor al resto de las especies, el cual fue más bajo en las estacas de las especies $P$. pennelli y P. smithii (figura 1d).

\section{Desarrollo de cada especie después de la siembra}

Las variables medidas en las estacas variaron de acuerdo con la especie de Passiflora (tabla 2, anexo 1) y para algunas se observaron correlaciones (anexo 2). En el ACP la primera dimensión estuvo correlacionada con el número de hojas $(0.884$.
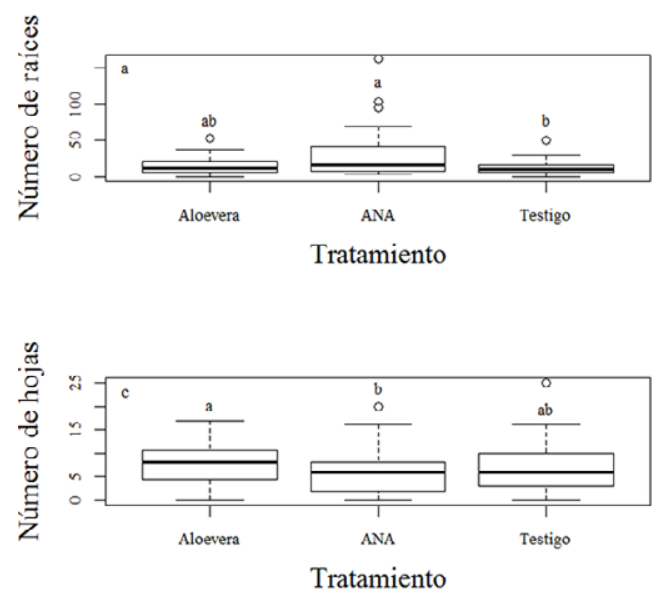

p-valor: <0.001), el número de zarcillos (0.874. p-valor: <0.001), la longitud del brote más largo (0.797. p-valor: $<0.001)$, el número de ramificaciones (0.501. p-valor: <0.001) y el número de brotes (0.390. p-valor: <0.001) que, de manera general, representan la construcción de tejidos aéreos en la etapa de desarrollo de la estaca; la segunda dimensión estuvo correlacionada con la longitud de la raíz más larga (0.745. p-valor: <0.001), la longitud del brote más largo (-0.410. p-valor: <0.001) y el número de raíces por estaca (-0.677. p-valor: $<0.001$ ), lo que representa la construcción de tejidos subterráneos. Ambas dimensiones acumularon una varianza de $55.95 \%$ (figura 2).

Las estacas de las especies $P$. pennelli y $P$. smithii tuvieron un desarrollo similar debido a que ocuparon un espacio semejante en el plano factorial, el cual indica valores bajos tanto en la dimensión uno (baja construcción de tejidos aéreos) como en la dimensión dos (baja construcción de tejidos subterráneos). Sin embargo, su desarrollo se caracterizó por la producción de un bajo número de raíces largas (figura 2, anexo 1). P. longipes mostró un mayor desarrollo de tejidos aéreos en comparación con los subterráneos, lo cual se evidencia en valores altos para la dimensión uno y bajos para la dos, puesto que en esta última primó la construcción de pocas raíces largas (figura 2, anexo 1 ). Para P. erythrophylla, la inversión en tejidos aéreos
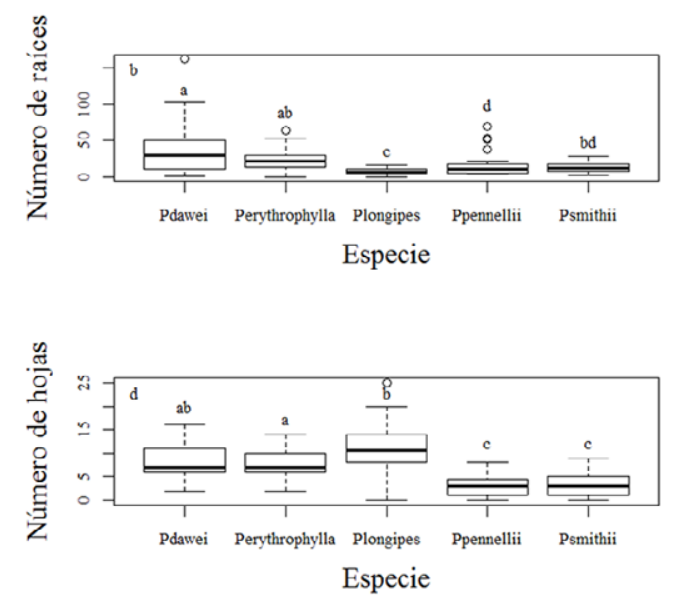

Figura 1. Número de raíces por tratamiento (a) y especie (b), y número de hojas por tratamiento (c) y especie (d). Letras no compartidas representan diferencias a un nivel de significación de 0.05. 

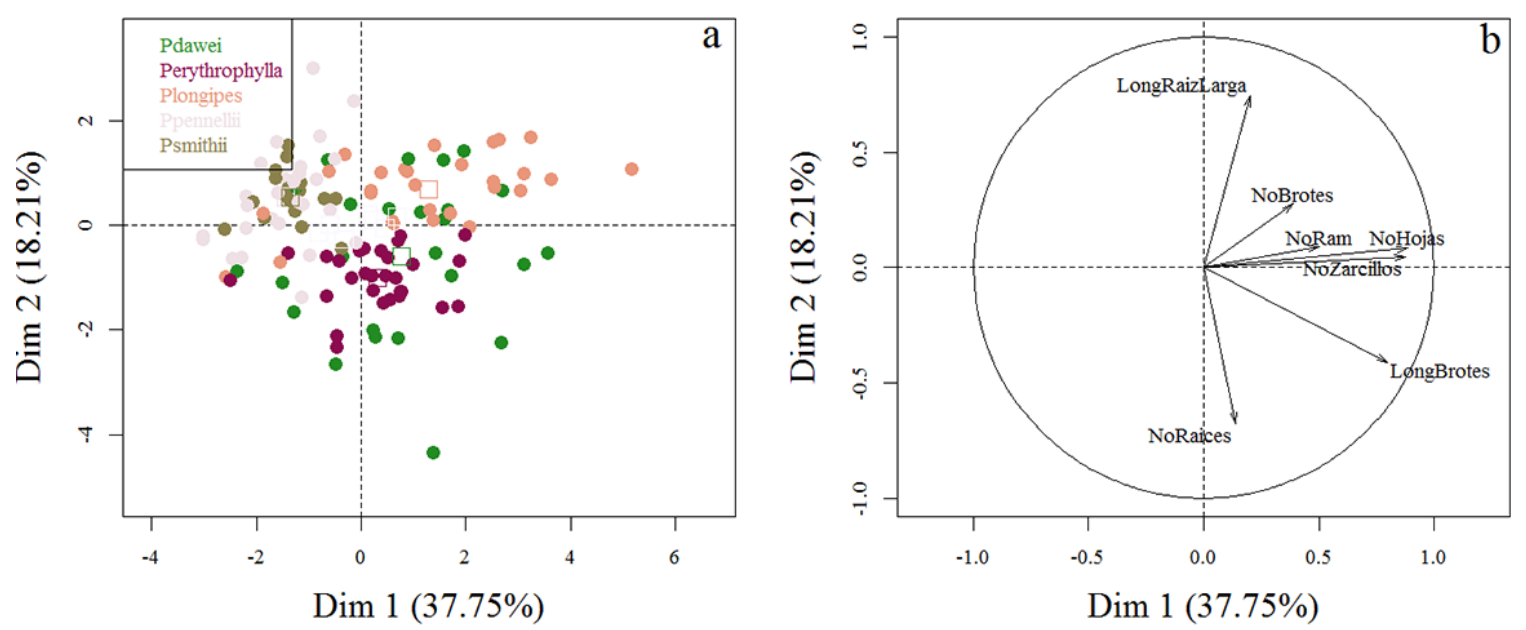

Figura 2. Análisis de componentes principales para las variables medidas en las estacas. Se presentan los individuos de cada especie (a) y las variables (b) sobre el plano factorial. LongBrotes: longitud del brote más largo (cm), LongRaizLarga: longitud de la raíz más larga $(\mathrm{cm})$, NoBrotes: número de brotes, NoHojas: número de hojas, NoRaices: número de raíces, NoRam: número de ramificaciones en una muestra de raíz de $3 \mathrm{~cm}$ y NoZarcillos: número de zarcillos.

fue mayor que en tejidos subterráneos dando prioridad a un mayor número de raíces cortas y brotes largos (figura 2, anexo 1). Finalmente, las estacas de $P$. dawei tuvieron la mayor variación en el plano factorial para ambas dimensiones, sin embargo, esta especie mostró crecimiento de brotes largos, de pocas hojas y raíces abundantes y largas durante su desarrollo (figura 2, anexo 1).

\section{DISCUSIÓN}

\section{Efecto de los tratamientos sobre las especies de Passiflora}

Durante el desarrollo del ensayo, se observó un ataque de hongos fitopatógenos presentes en el suelo de los géneros Fusarium y Verticillium, a lo cual se le atribuye la mortalidad de varias estacas. Específicamente, las estacas de $P$. smithii fueron las más vulnerables a este ataque, seguidas por las estacas de $P$. dawei, especialmente cuando se aplicaron cristales de sábila (tabla 2). De esta manera, la pasteurización, método empleado para la desinfección del sustrato en el que se evitó el uso de sustancias químicas (Hartmann y Kester, 1991), no fue muy efectivo para la eliminación de los hongos reportados, más aún cuando encontramos que varias especies propagadas en este estudio fueron susceptibles a este ataque, tal y como se ha reportado en la especie cultivada $P$. edulis $f$. flavicarpa (Campo-Arana y Vergara-Canedo, 2014). Así pues, es recomendable que adicional al proceso de pasteurización del sustrato y previo a la siembra de estacas, se realice una aplicación del hongo antagonista Trichoderma spp. para evitar el contagio de especies susceptibles (Martínez, Infante y Reyes, 2013), pues el medio en el cual se propagaron las estacas, donde hubo una alta humedad relativa, pudo ser un promotor importante para la proliferación de este tipo de hongos (Fischer y Rezende, 2008; Lima et al., 2019).

Por otra parte, los tratamientos aplicados a las estacas tuvieron efecto solamente sobre el número de raíces y el número de hojas (tabla 3, figura 1). Se observó que el ANA fue el mejor estimulante para la producción de un mayor número de raíces, aunque no difirió del tratamiento con cristales de sábila y el número de hojas fue el más bajo para las estacas tratadas con esta hormona. Esto señala que 
el ANA es un tratamiento efectivo, al igual que los cristales de sábila, pero puede promover una mayor producción de raíces en estacas como se ha evidenciado en otros estudios (Otahola y Vidal, 2010; Mendoza-F, Celis-F y Pachón-S, 2012; Minchala-Patiño et al., 2013; Morillo et al., 2016) aunque, como se observó en esta investigación, es posible que la producción de tejidos aéreos como las hojas disminuya debido a la compensación que la planta realiza al producir mayores raíces (Ryser y Lambers, 1995; Ryser y Eek, 2000). No obstante, esto último depende de las características de la especie y las estrategias empleadas en la etapa de crecimiento para el desarrollo de tejidos radiculares y aéreos (MacGregor, Deak, Ingram y Malamy, 2008). Pues, mientras $P$. longipes presentó el menor número de raíces y el mayor de hojas, este no fue el mismo patrón de compensación observado en el resto de especies: muchas raíces y hojas en $P$. dawei y $P$. erythrophylla y pocas raíces y hojas en $P$. pennelli y $P$. smithii; lo anterior indica que la magnitud del efecto del tratamiento varía de acuerdo con las características de la especie y sus necesidades durante el proceso de crecimiento. Además, es recomendable que se evalúen otros factores que pueden afectar el desarrollo radicular y aéreo de estas especies, pues existe evidencia que señala que el déficit de humedad, $\mathrm{pH}$ y la disponibilidad de nutrientes en el suelo influyen en el crecimiento vegetativo y en procesos fisiológicos asociados con la fotosíntesis (Menzel, Simpson y Dowling, 1986; Aiyelaagbe, Fagbayide y Makinde, 2005; Niwayama e Higuchia, 2018).

A pesar de que el número de raíces no fue estadísticamente distinto entre las estacas tratadas con ANA y cristales de sábila, se observó que en términos generales el ANA promovió los mayores valores (figura 1a). Adicionalmente, la mortalidad observada en estacas tratadas con cristales de sábila fue mayor (35\%) frente a las tratadas con ANA $(23.3 \%)$, lo cual indica que a pesar de que ambos tratamientos fueron eficaces en el enraizamiento, el ANA pudo prevenir la mortalidad de un mayor número de estacas. Este resultado contradice las expectativas frente a la experiencia del JBB en el empleo de cristales de sábila para propagar estacas de especies de Passiflora, pues comúnmente se consideraba el más efectivo, pero no se había probado experimentalmente. Lo anterior no quiere decir que los cristales de sábila no son un buen tratamiento, porque, además de que es una alternativa económica, se ha probado que tienen efectos importantes en el enraizamiento de otras especies e incluso mayores que las hormonas sintéticas (Rodríguez y Hechevarría, 2004; Giraldo et al., 2009; Borges et al., 2016), solo que en esta investigación se demostró que el ANA tuvo ciertas ventajas sobre el enraizamiento de especies de Passiflora.

Si bien los métodos de propagación asexual convencionales se emplean con menos frecuencia recientemente, y en el caso de especies de Passiflora el método más estudiado es la micropropagación (Isutsa, 2004; Carneiro y Sampaio, 2005; Yockteng et al., 2011; Ozarowski y Thiem, 2013; Shekhawat, Kannan, Manokari y Ravindran, 2015; Castro et al., 2016; Pacheco et al., 2016), los resultados de esta investigación representan un aporte importante a las necesidades que actualmente demanda la conservación ex situ de estas especies en los jardines botánicos (García et al., 2010; Samper y García, 2001). Específicamente, nuestros resultados aportan en la mejora del conocimiento y el proceso de propagación de especies de Passiflora endémicas y amenazadas en los Andes colombianos empleando métodos fáciles de aplicar y evaluar.

\section{Desarrollo de cada especie después de la siembra}

Se encontró que cada especie presentó un desarollo particular que influyó en la mayoría de variables medidas tanto en los tejidos aéreos como subterráneos (tabla 2, figura 2), lo cual es una respuesta independiente al tratamiento aplicado y al propósito buscado con el mismo. Específicamente, encontramos especies de bajo desarrollo de tejidos aéreos, de pocas, pero largas raíces ( $P$. pennelli y $P$. smithii), especies de alto desarrollo de tejidos aéreos, pero con un desarrollo radicular bajo 
en cantidad o longitud de raíces $(P$. longipes y $P$. erythrophylla) y una especie que presentó estacas de desarrollo variable pero similar tanto en los tejidos aéreos como subterráneos ( $P$. dawei).

Es posible que el efecto de las condiciones ambientales experimentales haya tenido un efecto sobre la respuesta en el desarrollo de las estacas para cada especie con respecto a las condiciones de hábitat natural (Leimu y Fischer, 2008). Por ejemplo, P. pennelli y P. smithii son especies que crecen en altitudes bajas (500 a 2000 m) en comparación con $P$. longipes y $P$. erythrophylla que habitan zonas más altas (1600 a 3500 m) (Ocampo et al., 2007; Bernal et al., 2015), lo cual podría explicar que las primeras hayan tenido un bajo y las segundas un alto desarrollo de tejidos aéreos que fue favorecido por el sitio en el que fueron propagadas sus estacas, a $2600 \mathrm{~m}$ de altitud y con temperaturas que no superaron los $22^{\circ} \mathrm{C}$. Sin embargo, este posible efecto debe ser evaluado en el futuro para determinar el impacto de condiciones ex situ sobre el crecimiento de especies de Passiflora.

A su vez, es posible que la diferenciación en la inversión de tejidos radiculares y aéreos haya sido causa de la respuesta fenotípica de las especies ante la modificación de las condiciones ambientales naturales a artificiales (Ryser y Lambers, 1995; Ryser y Eek, 2000). Esto ocurre especialmente en las especies de altitudes bajas, $P$. pennelli y $P$. smithii que, comparadas con $P$. longipes y $P$. erythrophylla, tienen tallos más gruesos y hojas más gruesas y lobuladas con un costo y tiempo mayor de construcción, por lo cual las condiciones ambientales de propagación diferentes a su hábitat natural, pudieron afectar el desarrollo de tejidos aéreos y fomentar el de tejidos radiculares (Ryser y Eek, 2000; Leimu y Fischer, 2008). Por el contrario, la respuesta fenotípica de las estacas de $P$. dawei fue particular, pues su variabilidad en el desarrollo y la alta producción casi similar de tejidos aéreos y radiculares en condiciones distintas a su hábitat natural muestra que este método de propagación hace a la especie altamente competitiva (Ryser y Lambers, 1995) con un buen potencial de adaptación en etapa juvenil para la posterior conservación ex situ.

En vista de esto, se deben considerar las particularidades de cada especie y sus preferencias fisiológicas de desarrollo al momento de definir un tratamiento de propagación y el objetivo que se persigue con este. Para esto, se ha demostrado la influencia de hormonas en el desarrollo de distintos tejidos de las plantas, entre estas, se reconoce el efecto de las auxinas sobre el desarrollo radicular (Péret et al., 2009; Lavenus et al., 2013), las citoquininas en la formación de nuevos brotes, diferenciación de tallos y consecuente reducción de dominancia apical y las giberelinas que, al contrario, favorecen la dominancia apical y el crecimiento en altura (Jordán y Casaretto, 2007; Taiz, Zeiger, Moller y Murphy, 2017). De manera que, de acuerdo con el objetivo de propagación, es recomendable que se evalúe el empleo de citoquininas y giberelinas en estacas de especies caracterizadas por bajo desarrollo de tejidos aéreos como fue el caso de estacas de P. pennelli y P. smithii con el fin de identificar posibles mejoras en el desarrollo de estos tejidos. Además, se sugiere comparar distintas concentraciones de auxinas en estacas de especies caracterizadas por bajo desarrollo de tejidos radiculares como $P$. longipes y $P$. erythrophylla con el propósito de mejorar su desarrollo radicular durante el proceso de propagación.

\section{CONCLUSIONES}

Los tratamientos empleados sobre las estacas de especies de Passiflora (ANA y cristales de sábila) tuvieron efecto en el número de raíces y hojas, pero la magnitud del efecto fue distinta para cada especie. Las estacas tratadas con ANA presentaron un mayor número de raíces que las tratadas con cristales de sábila y, adicionalmente, mostraron la menor mortalidad. Así pues, se recomienda el empleo de esta hormona para la propagación de estacas de Passiflora cuando se requiere aumentar la producción de raíces, pero se sugiere también el empleo de cristales de sábila como una alternativa mucho más económica y natural que 
promueve resultados similares en el enraizamiento de estacas. Conviene subrayar que es fundamental la previa desinfección del sustrato y la aplicación de hongos antagonistas de Fusarium spp. y Verticillium spp., los cuales infectaron las estacas de las especies evaluadas en este estudio y se identificó a $P$. smithii y $P$. dawei como las más vulnerables.

Durante el proceso de desarrollo de las estacas, P. pennelli y $P$. smithii se caracterizaron por un bajo desarrollo de tejidos aéreos con pocas raíces largas, P. longipes y P. erythrophylla por un alto desarrollo de tejidos aéreos y bajo desarrollo radicular en cantidad y longitud de raíces respectivamente, lo cual pudo deberse a diferencias en la plasticidad de cada especie como respuesta a las condiciones artificiales impuestas durante la propagación y que tuvieron diferencias con la zona de hábitat natural. Debido al desarrollo similar de tejidos aéreos y radiculares de las estacas de $P$. dawei y a su variabilidad entre individuos, el método de propagación asexual empleado hace que la especie sea la más competitiva en su estado juvenil entre las cinco evaluadas con un buen potencial de adaptación para la posterior conservación ex situ.

Para definir tratamientos de propagación, se recomienda considerar las características y requerimientos particulares de cada especie con el fin de cubrir sus necesidades en la etapa de desarrollo de los propágulos. Para la propagación de estacas de P. pennelli y P. smithii es recomendable realizar ensayos con citoquininas y giberelinas con el fin de mejorar el bajo desarrollo de tejidos aéreos que presentaron. En el caso de $P$. longipes y P. erythrophylla se sugiere evaluar si diferencias en la concentración de auxinas mejoran el bajo desarrollo radicular observado en este estudio, de manera que se optimice el proceso de desarrollo de las estacas.

\section{AGRADECIMIENTOS}

Esta investigación fue realizada en la línea de colecciones vivas, sublínea Cepac-Passifloraceae, de la subdirección científica bajo los contratos de prestación de servicios 296-2018 y 624-2018 con el Jardín Botánico de Bogotá José Celestino Mutis. Los autores expresan agradecimientos especiales al operario César Molina y al profesional Kristian Rubiano por su apoyo en el montaje del ensayo y medición de variables, al técnico Álvaro Díaz por su apoyo en la identificación de los hongos fitopatógenos que afectaron las estacas de Passiflora, y al operario Miguel Quintero por facilitar el espacio para el montaje del ensayo dentro del Jardín.

\section{CONFLICTO DE INTERESES}

Los autores declaran no tener conflicto de intereses.

\section{CONTRIBUCIÓN POR AUTOR}

B.L. C-R. y G. M-L. planearon y diseñaron la investigación, coordinaron y llevaron a cabo la investigación en el terreno, analizaron los datos y redactaron el manuscrito.

Ambos autores contribuyeron a la discusión y comentaron los borradores..

\section{REFERENCIAS}

Aiyelaagbe, I. O. O., Fagbayide, J. A. y Makinde, A. I. (2005). Agriculture Effects of $\mathrm{N}$ fertilization on the vegetative growth of passion fruit (Passiflora edulis f. flavicarpa) seedlings. Journal of Food, Agriculture \& Environment, 3(3 y 4), 62-64.

Armenteras, D. y Rodríguez, N. (2014). Dinámicas y causas de deforestación en bosques de Latino América: una revisión desde 1990. Colombia Forestal, 17(2), 233-246.

https://doi.org/10.14483/udistrital.jour.colomb.for.2014.2.a07

Bernal, R., Gradstein, S. y Celis, M. (2015). Catálogo de plantas y líquenes de Colombia. Recuperado de http://catalogoplantasdecolombia.unal.edu.co/es/

Borges, J. A., León, M., Marturet, E. y Barrios, M. (2016). Fitoestimulación en estacas de morera 
(Morus alba L.) mediante extractos vegetales. Bioagro, 28(3), 215-219.

https://doi.org/10.1007/s00192-013-2125-8

Campo-Arana, R. O. y Vergara-Canedo, L. S. (2014). Evaluación de la resistencia de la población de maracuyá amarillo (Passiflora edulis f. flavicarpa. Degener), de San Isidro, Córdoba, a la marchitez vascular (Fusarium spp.). Fitopatología Colombiana, 38(2), 39-42.

https://doi.org/10.18257/raccefyn.5

Carneiro, M. L. y Sampaio, M. (2005). 17.1 Passiflora spp. Passionfruit. En R. Litz (ed.), Biotechnology in Agriculture Series No. 29 Biotechnology of fruit and nut crops (pp. 436-453). Cambridge, EE. UU.: CAB International.

https://doi.org/10.1079/9780851996622.0436

Castro, J. A., Oliveira, E. J., Jesus, O. N., Soares, T. L. y Margarido, G. R. A. (2016). Molecular markers for conservation genetic resources of four Passiflora species. Scientia Horticulturae, 212, 251-261.

https://doi.org/10.1016/j.scienta.2016.10.005

Dhawan, K., Dhawan, S. y Sharma, A. (2004). Passiflora: A review update. Journal of Ethnopharmacology, 94, 1-23.

https://doi.org/10.1016/j.jep.2004.02.023

Faleiro, F. G., Junqueira, N. T. V. y Braga, M. F. (2005). Maracujá germoplasma e melhoramento genético. Planaltina-DF, Brasil: Empresa Brasileira de Pesquisa Agropecuária Embrapa Cerrados, Ministério da Agricultura, Pecuária e Abastecimento.

https://doi.org/10.17138/tgft(1)95-96

Fischer, I. H., y Rezende, J. A. M. (2008). Diseases of Passion Flower (Passiflora spp.). Pest Technology, 2(1), 1-9.

Fox, J. y Weisberg, S. (2011). An $\{R\}$ Companion to Applied Regression, Second Edition. Thousand Oaks CA: Sage. Recuperado de

http://socserv.socsci.mcmaster.ca/jfox/Books/ Companion

García, H., Moreno, L., Londoño, C. y Sofrony, C. (2010). Estrategia nacional para la conservación de plantas actualización de los antecedentes normativos y políticos, y revisión de avances. Bogotá: Instituto de
Investigación de Recursos Biológicos Alexander Von Humboldt y Red Nacional de Jardines Botánicos.

https://doi.org/10.21068/d100818oc

Giraldo, L., Ríos, H. y Polanco, M. (2009). Efecto de dos enraizadores en tres especies forestales promisorias para la recuperación de suelos. Revista de Investigación Agraria y Ambiental RIAA, 0(1), 41-47.

https://doi.org/10.22490/21456453.1966

Harrell, F. Y Dupont, C. (2018). Hmisc: Harrell MisceIlaneous. $R$ package version 4.1-1. Recuperado de

https://cran.r-project.org/package=Hmisc

Hartmann, H. y Kester, D. (1991). Propagación de plantas principios y prácticas. México D.F.: Compañía Editorial Continental.

Instituto de Hidrología, Meteorología y Estudios Ambientales (Ideam). (2018). 17 Boletín de detección temprana de deforestación cuarto trimestre octubre - diciembre 2018. Bogotá, D.C., Colombia: Instituto de Hidrología, Meteorología y Estudios Ambientales (Ideam). Recuperado de:

http://documentacion.ideam.gov.co/openbiblio/bvirtual/023856/17_BoletinAT-D.pdf

Isutsa, D. K. (2004). Rapid micropropagation of passion fruit (Passiflora edulis Sims.) varieties. Scientia Horticulturae, 99(3-4), 395-400.

https://doi.org/10.1016/j.scienta.2003.08.002

Jordán, M. y Casaretto, J. (2007). Hormonas y reguladores del crecimiento: auxinas, giberelinas y citocininas. En F. Saqueo y L. Cardemil (eds.), Fisiología Vegetal (pp. 1-28). La Serena, Chile: Ediciones Universidad de La Serena.

Killip, E. (1938). The American Species of Passifloraceae Field Museum of Natural History Publication. Botanical Series, 19(1,2), 1-613.

Lavenus, J., Goh, T., Roberts, I., Guyomarc'h, S., Lucas, M., De Smet, I.,... Laplaze, L. (2013). Lateral root development in Arabidopsis: Fifty shades of auxin. Trends in Plant Science, 18(8), 1360-1385. https:// doi.org/10.1016/j.tplants.2013.04.006

Lê, S., Josse, J. y Husson, F. (2008). FactoMineR: An R Package for Multivariate Analysis. Journal of Statistical Software, 25(1), 1-18.

https://doi.org/10.1016/j.envint.2008.06.007 
Leimu, R. y Fischer, M. (2008). A meta-analysis of local adaptation in plants. PLOS ONE, 3(12), 1-8.

https://doi.org/10.1371/journal.pone.0004010

Lima, L. K. S., de Jesus O. N., Soares, T. L., de Oliveira, S. A. S., Haddad, F. y Girardi, E. A. (2019). Water deficit increases the susceptibility of yellow passion fruit seedlings to Fusarium wilt in controlled conditions. Scientia Horticulturae, 243, 609-621.

https://doi.org/10.1016/j.scienta.2018.09.017

MacGregor, D. R., Deak, K. I., Ingram, P. A. y Malamy, J. E. (2008). Root System Architecture in Arabidopsis Grown in Culture Is Regulated by Sucrose Uptake in the Aerial Tissues. The Plant Cell, 20, 2643-2660.

https://doi.org/10.1105/tpc.107.055475

Mangiafico, S. (2018). rcompanion: Functions to Support Extension Education Program Evaluation. $\mathrm{R}$ package version 2.0.3. Recuperado de:

https://cran.r-project.org/package=rcompanion $\% 0 \mathrm{~A} \% 0 \mathrm{~A}$

Martínez, B., Infante, D. y Reyes, Y. (2013). Trichoderma spp. y su función en el control de plagas en los cultivos. Revista de Protección Vegetal, 28(1), 1-11.

Mendoza-F, C., Celis-F, A. y Pachón-S, M. E. (2012). Evaluation of Propagation Methods by Seeds and Cuttings in Piper aduncum (Piperaceae). Acta Horticulture, 964, 129-134.

https://doi.org/https://doi.org/10.17660/ActaHortic. 2012.964.16

Menzel, C. M., Simpson, D. R. y Dowling, A. J. (1986). Water relations in passionfruit: Effect of moisture stress on growth, flowering and nutrient uptake. Scientia Horticulturae, 29, 239-249.

https://doi.org/10.1016/0304-4238(86)90067-1

Minchala-Patiño, J., Eras Guaman, V. H., Muñoz Chamba, L., Yaguana Arevalo, M., Poma Angamarca, R. y Delgado Paredes, G. (2013). Propagación sexual y asexual de cuatro especies forestales nativas y promisorias de la Región Sur del Ecuador. Cedamaz, $3(1), 5-15$.

Montgomery, D. (2004). Diseño y análisis de experimentos. México D.F.: Editorial Limusa, S.A. de C.V. Grupo Noriega Editores.

Morillo, L. F., Eras, V. H., Moreno, J., Minchala, J., Muñoz, L., Yaguana, M.,... Sinche, M. (2016). Estudio fenológico y propagación de Bursera graveolens (Kunth) Triana \& Planch, en la comunidad de Malvas, cantón Zapotillo, provincia de Loja. Bosques Latitud Cero, 6(2), 1-15.

Niwayama, S. y Higuchia, H. (2018). Effects of soil pH on the root growth of passion fruit and the mechanism of mineral uptake. Acta Horticulturae, 1217, 111-119.

https://doi.org/10.17660/ActaHortic.2018.1217.14

Ocampo, J., D'Eeckenbrugge, G. C., Restrepo, M., Jarvis, A., Salazar, M. y Caetano, C. (2007). Diversity of Colombian Passifloraceae: biogeography and an updated list for conservation. Biota Colombiana, 8(1), 179-207.

https://doi.org/10.21068/bc.v8i1.181

Ocampo, J., d'Eeckenbrugge, G. C. y Jarvis, A. (2010). Distribution of the genus Passiflora L. Diversity in Colombia and its potential as an indicator for biodiversity management in the coffee growing zone. Diversity, 2(11), 1158-1180.

https://doi.org/10.3390/d2111158

Ocampo, J. y Wyckhuys, K. A. G. (2012). Tecnología para el cultivo de la Gulupa en Colombia (Passiflora edulis f. edulis Sims). Bogotá D.C., Colombia: Centro de Bio-Sistemas de la Universidad Jorge Tadeo Lozano, Centro Internacional de Agricultura Tropical-CIAT y Ministerio de Agricultura y Desarrollo Rural.

https://doi.org/10.17138/tgft(2)130-132

Ogle, D., Wheeler, P. y Dinno, A. (2018). FSA: Fisheries Stock Analysis. R package version 0.8.22. Recuperado de: https://github.com/droglenc/FSA

Oliva-Cruz, C. A. (2005). Efecto de fitoreguladores enraizantes y la temperatura en el enraizamiento de estacas de Myrciaria dubia (HBK) MC Vaugh, camu camu arbustivo, en Ucayali-Perú. Folia Amazónica, 14(2), 19-25.

https://doi.org/10.24841/fa.v14i2.141

Otahola, V. y Vidal, G. (2010). Efecto de las características de la estaca y la utilización de ANA en la propagación de parchita (Passiflora edulis f. flavicarpa Deg.). Revista Científica UDO Agrícola, 10(1), 29-35.

https://doi.org/10.21640/ns.v8i17.497 
Ozarowski, M. y Thiem, B. (2013). Progress in micropropagation of Passiflora spp. to produce medicinal plants: A mini-review. Brazilian Journal of Pharmacognosy, 23(6), 937-947.

https://doi.org/10.1590/S0102-695X2013000600011

Pacheco, G., Simão, M. J., Vianna, M. G., Garcia, R. O., Vieira, M. L. C. y Mansur, E. (2016). In vitro conservation of Passiflora-A review. Scientia Horticulturae, 211, 305-311.

https://doi.org/10.1016/j.scienta.2016.09.004

Parra, M., Carranza, C., Cárdenas, J. y Miranda, D. (2010). Memorias Primer Congreso Latinoamericano de Passiflora. Neiva, Huila, Colombia: Corporación Centro de Investigación para la Gestión Tecnológica de Passiflora del Departamento del Huila.

https://doi.org/10.22507/pml.v11n2a9

Péret, B., De Rybel, B., Casimiro, I., Benková, E., Swarup, R., Laplaze, L.,... Bennett, M. J. (2009). Arabidopsis lateral root development: an emerging story. Trends in Plant Science, 14(7), 399-408.

https://doi.org/10.1016/j.tplants.2009.05.002

Pérez, R., Reynel, C. y Manta, M. (2002). Dendrología y propagación vegetativa de Acacia horrida ("huaranguillo") mediante estacas inducidas en tres sustancias enraizantes, usando sustratos. Ecología Aplicada, 1(1), 9-12.

https://doi.org/10.21704/rea.v1i1-2.223

R Core Team. (2018). R: A language and environment for statistical computing. Vienna: R Foundation for Statistical Computing. Recuperado de

https://www.r-project.org/

Resolución $\mathbf{N}^{\circ} \mathbf{2 4 9}$ Por la cual se actualiza y adopta el Plan de Investigaciones del Jardín Botánico de Bogotá José Celestino Mutis 2018-2028. Bogotá D.C., Colombia, 27 de julio de 2018.

https://doi.org/10.17151/luaz.2017.45.11

Rodríguez, H. y Hechevarría, I. (2004). Efectos estimulantes del crecimiento de extractos acuosos de plantas medicinales y gel de Aloe vera (L.) N. L. Burm. Revista Cubana de Plantas Medicinales, 9(2), 1-8. https://doi.org/10.18636/ribd.v30i1.296
Rubiano, K., Clerici, N., Norden, N. y Etter, A. (2017). Secondary Forest and Shrubland Dynamics in a $\mathrm{Hi}$ ghly Transformed Landscape in the Northern Andes of Colombia (1985-2015). Forest, 8(6), 216.

https://doi.org/10.3390/f8060216

Ryser, P. y Lambers, H. (1995). Root and leaf attributes accounting for the performance of fast- and slowgrowing grasses at different nutrient supply. Plant and Soil, 170(2), 251-265.

https://doi.org/10.1007/BF00010478

Ryser, P. y Eek, L. (2000). Consequences of phenotypic plasticity vs. interspecific differences in leaf and root traits for acquisition of aboveground and belowground resources. American Journal of Botany, 87(3), 402-411.

https://doi.org/10.2307/2656636

Samper, C. y García, H. (2001). Estrategia Nacional para la Conservación de Plantas. Villa de Leyva, Colombia: Instituto de Investigación de Recursos Biológicos Alexander von Humboldt.

https://doi.org/10.21068/d100818oc

Shekhawat, M. S., Kannan, N., Manokari, M. y Ravindran, C. P. (2015). In vitro regeneration of shoots and ex vitro rooting of an important medicinal plant Passiflora foetida L. through nodal segment cultures. Journal of Genetic Engineering and Biotechnology, 13(2), 209-214.

https://doi.org/10.1016/j.jgeb.2015.08.002

Taiz, L., Zeiger, E., Moller, I. y Murphy, A. (2017). Fisiologia e Desenvolvimento Vegetal. São Paulo, Brazil: Artmed Editora Ltda.

Ulmer, T. y MacDougal, J. (2004). Passiflora: Passionflowers of the world. Editorial Timber Press.

Yockteng, R., D'Eeckenbrugge, G. C. y Souza-Chies, T. (2011). Chapter 7 Passiflora. En C. Kole (ed.), Wild Crop Relatives: Genomic and Breeding Resources Tropical and Subtropical Fruits (pp. 129171). Clemson, USA: Springer-Verlag Berlin Heidelberg.

https://doi.org/10.1007/978-3-642-20447-0_7 


\section{ANEXOS}

Anexo 1. Resultados del post-hoc Dun para los factores que tuvieron un efecto sobre las variables evaluadas en estacas de Passiflora. Letras no compartidas representan diferencias a un nivel de significación de 0.05.

\begin{tabular}{cccc}
\hline Factor & Nivel & Mediana & Grupo \\
\hline \multirow{4}{*}{ Tratamiento } & Número de raíces & \\
& Cristales de & 12 & $\mathrm{ab}$ \\
& sábila & & \\
& ANA & 16 & $\mathrm{a}$ \\
& Testigo & 11 & $\mathrm{~b}$ \\
Especie & P. dawei & 30 & $\mathrm{a}$ \\
& P. erythrophylla & 21 & $\mathrm{ab}$ \\
& P. longipes & 6 & $\mathrm{C}$ \\
& P. pennellii & 10 & $\mathrm{~d}$ \\
& P. smithii & 12 & $\mathrm{~b} \mathrm{~d}$
\end{tabular}

Número de ramificaciones

\begin{tabular}{|c|c|c|c|}
\hline Factor & Nivel & Mediana & Grupo \\
\hline \multicolumn{4}{|c|}{ Número de raíces } \\
\hline \multicolumn{4}{|c|}{ Longitud del brote más largo $(\mathrm{cm})$} \\
\hline & P. dawei & 23.00 & $a b$ \\
\hline \multirow{4}{*}{ Especie } & P. erythrophylla & 31.80 & $\mathrm{a}$ \\
\hline & P. longipes & 19.00 & $b$ \\
\hline & P. pennellii & 3.00 & c \\
\hline & P. smithii & 1.85 & c \\
\hline \multicolumn{4}{|c|}{ Número de hojas } \\
\hline \multirow{5}{*}{ Tratamiento } & $\begin{array}{c}\text { Cristales de } \\
\text { sábila }\end{array}$ & 8 & a \\
\hline & ANA & 6 & $\mathrm{~b}$ \\
\hline & Testigo & 6 & $a b$ \\
\hline & P. dawei & 7.0 & $a b$ \\
\hline & P. erythrophylla & 7.0 & $\mathrm{a}$ \\
\hline \multirow[t]{3}{*}{ Especie } & P. longipes & 10.5 & b \\
\hline & P. pennellii & 3.0 & c \\
\hline & P. smithii & 3.0 & c \\
\hline \multicolumn{4}{|c|}{ Número de zarcillos } \\
\hline \multirow{5}{*}{ Especie } & P. dawei & 5 & $\mathrm{a}$ \\
\hline & P. erythrophylla & 1 & $\mathrm{~b}$ \\
\hline & P. longipes & 6 & $\mathrm{a}$ \\
\hline & P. pennellii & 0 & c \\
\hline & P. smithii & 0 & c \\
\hline
\end{tabular}

Número de brotes

$\begin{array}{ccc}\text { P. dawei } & 4 & \mathrm{ab} \\ \text { P. erythrophylla } & 6 & \mathrm{a} \\ \text { P. longipes } & 6 & \mathrm{a} \\ \text { P. pennellii } & 4 & \mathrm{~b} \\ \text { P. smithii } & 6 & \mathrm{a}\end{array}$

Longitud de la raíz más larga $(\mathrm{cm})$

$\begin{array}{cccc} & \text { P. erythrophylla } & 15.00 & \mathrm{~b} \\ \text { Especie } & \text { P. longipes } & 26.35 & \mathrm{a} \\ & \text { P. pennellii } & 25.40 & \mathrm{a} \\ & \text { P. smithii } & 25.35 & \mathrm{a}\end{array}$

$\mathrm{b}$
$\mathrm{a}$
$\mathrm{b}$
$\mathrm{a}$
$\mathrm{a}$
$\mathrm{b}$
$\mathrm{a}$
$\mathrm{a}$
$\mathrm{a}$

\begin{tabular}{cccc} 
& P. dawei & 1.0 & $\mathrm{ab}$ \\
Especie & P. erythrophylla & 1.0 & $\mathrm{a}$ \\
& P. longipes & 1.5 & $\mathrm{~b}$ \\
& P. pennellii & 1.0 & $\mathrm{a}$ \\
& P. smithii & 1.0 & $\mathrm{ab}$ \\
\hline
\end{tabular}


Anexo 2. Correlaciones de Spearman entre variables evaluadas en estacas de Passiflora. En negrilla las correlaciones que fueron significativas a un nivel de 0.05 .

\begin{tabular}{|c|c|c|c|c|c|c|c|}
\hline Variables & $\begin{array}{l}\text { Número } \\
\text { de raíces }\end{array}$ & $\begin{array}{c}\text { Número de } \\
\text { ramificaciones }\end{array}$ & $\begin{array}{l}\text { Longitud de la } \\
\text { raíz más larga }\end{array}$ & $\begin{array}{l}\text { Número } \\
\text { de brotes }\end{array}$ & $\begin{array}{l}\text { Longitud } \\
\text { de brotes }\end{array}$ & $\begin{array}{l}\text { Número } \\
\text { de hojas }\end{array}$ & $\begin{array}{c}\text { Número de } \\
\text { zarcillos }\end{array}$ \\
\hline \multicolumn{8}{|l|}{ Matriz de correlaciones } \\
\hline Número de raíces & 1 & 0.130 & -0.080 & -0.070 & 0.340 & 0.120 & 0.110 \\
\hline $\begin{array}{l}\text { Número de ramifica- } \\
\text { ciones }\end{array}$ & 0.130 & 1 & 0.070 & 0.060 & 0.320 & 0.230 & 0.200 \\
\hline $\begin{array}{l}\text { Longitud de la raíz más } \\
\text { larga }\end{array}$ & -0.080 & 0.070 & 1 & 0.110 & -0.130 & 0.120 & 0.190 \\
\hline Número de brotes & -0.070 & 0.060 & 0.110 & 1 & 0.160 & 0.400 & 0.220 \\
\hline Longitud de brotes & 0.340 & 0.320 & -0.130 & 0.160 & 1 & 0.730 & 0.710 \\
\hline Número de hojas & 0.120 & 0.230 & 0.120 & 0.400 & 0.730 & 1 & 0.750 \\
\hline Número de zarcillos & 0.110 & 0.200 & 0.190 & 0.220 & 0.710 & 0.750 & 1 \\
\hline \multicolumn{8}{|c|}{ Valores $p$ asintóticos (significancia: 0.05 ) } \\
\hline Número de raíces & & 0.132 & 0.351 & 0.405 & $<0.001$ & 0.183 & 0.212 \\
\hline $\begin{array}{l}\text { Número de ramificacio- } \\
\text { nes en una muestra de } \\
\text { raíz de } 3 \mathrm{~cm}\end{array}$ & 0.1316 & & 0.405 & 0.487 & $<0.001$ & 0.008 & 0.022 \\
\hline $\begin{array}{l}\text { Longitud de la raíz más } \\
\text { larga }\end{array}$ & 0.3508 & 0.405 & & 0.238 & 0.132 & 0.191 & 0.033 \\
\hline Número de brotes & 0.4045 & 0.487 & 0.238 & & 0.066 & $<0.001$ & 0.012 \\
\hline Longitud de brotes & $<0.001$ & $<0.001$ & 0.132 & 0.066 & & $<0.001$ & $<0.001$ \\
\hline Número de hojas & 0.1825 & 0.008 & 0.191 & $<0.001$ & $<0.001$ & & $<0.001$ \\
\hline Número de zarcillos & 0.2116 & 0.022 & 0.033 & 0.012 & $<0.001$ & $<0.001$ & \\
\hline
\end{tabular}

